68

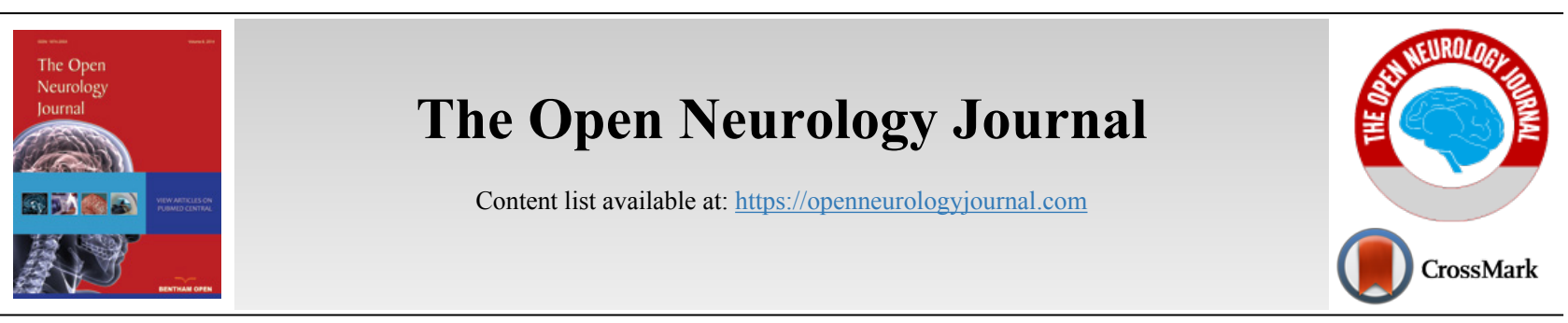

CASE REPORT

\title{
PET CT Brain for the Differential Diagnosis of Dementia -Indian Case Reports
}

\author{
Aman Gupta,"* and Aashish Gambhir ${ }^{2}$ \\ ${ }^{\prime}$ Amity Institute of Neuropsychology and Neurosciences, Amity University Uttar Pradesh, Sector-125, Noida-201313, India \\ ${ }^{2}$ Department of Nuclear Medicine and PET-CT, Yashoda Hospital and Research Centre Ghaziabad Uttar Pradesh 201001 Delhi NCR - Uttar \\ Pradesh 201001
}

\begin{abstract}
:
India has been termed as Diabetic hub of the globe. Along with other complications, one of the underestimated complication is cognitive decline and memory loss associated with poor diabetes control. In the Indian context, structural imaging like MRI-Magnetic Resonance imaging is more commonly used in Neurological disorders such as stroke, head injury, Functional imaging of the human brain has been underutilized in the Indian scenario. One such technique is PET CT which has been typically used as a cancer biomarker in India. By virtue of current case study, we propose i) association between poor control of Diabetes and poor cognition ii) role of PET CT brain in differential diagnosis of Dementia. We present two case reports providing strong findings for utilization of PET CT brain in Dementia protocols.
\end{abstract}

Keywords: Diabetes, Memory, Dementia, Cognition, Alzheimer's Disease, PET CT.

\begin{tabular}{|l|l|l|l|}
\hline Article History & Received: December 17, 2019 & Revised: March 16, 2020 & Accepted: March 18, 2020
\end{tabular}

\section{INTRODUCTION}

Clinical diagnosis of Dementia has always been challenging for the neurologists. Things become more difficult when one has to establish the differential diagnosis of Dementia. Globally, functional MRI is quite well known as a diagnostic tool; however, in Indian scenario, functional MRI centres are very limited. Considering the importance of functional imaging in differential diagnosis of dementia, authors chose to study the role of PET CT brain in dementia diagnosis. India is the hub for type 2 diabetes patients. The starting age of type 2 diabetes is 35 years and onwards. Our proposal is that diabetes can contribute to dementia and cognitive decline along with other regular dementia causing factors. In India, PET CT is primarily used for the purpose of cancer detection. Since there is already PET CT infrastructure available in India, use of existing PET CT in differential diagnosis of dementia can be of great advantage . Cognitive batteries can help in determining the cognitive decline, however the differential diagnosis of dementia may not be possible with the use of cognitive batteries. Current case study demonstrates utility of Positron emission tomography-computed tomography (PET CT) in brain for localizing areas of brain hypometabolism in the differential diagnosis of

\footnotetext{
* Address correspondence to this author at the Amity Institute of Neurosciences, Amity University Uttar Pradesh, Noida, India, Previously At, Visiting Fellowship Functional MRI, Harvard Medical School, USA, Diplomate in Sleep Medicine, University of Oxford, London, UK; Tel.: +911294013135, M:919953270158;

E-mail:Gupta.draman@gmail.com
}

Dementia. PET CT brain findings were suggestive of a specific pattern of reduced ${ }^{18} \mathrm{~F}$-Fludeoxyglucose $\left({ }^{18} \mathrm{~F}\right.$-FDG) uptake indicating Alzheimer's type Dementia. This case study can be instrumental to set up a dedicated protocol employing PET CT brain for differential diagnosis of dementia.

\section{CASE REPORT/CASE PRESENTATION}

Female type 2 Diabetic patient aged 74 years presented in outpatient department of a hospital with chief complaint of cognitive decline and memory loss over the last six months. Her medical history was significant for poor Diabetes control over the last 10 years. In the last three months, she had consulted different Neurologists and has been provisionally diagnosed as fronto-temporal Dementia without any radiological markers like Functional Magnetic Resonance Imaging (MRI), PET CT, etc. On physical examination, the patient was alert and conscious. Pupils reacted to light and eye muscle movements were intact.

Cranial nerve examination was found to be normal. Her muscle power for all four limbs was also normal. As the patient came with chief complaint of cognitive decline, patient was screened with validated cognitive batteries: General Practitioner Assessment of Cognition (GPCOG) score $=3$, Attendant Informant Tool (AI) score $=2$, Memory Impairment Screen (MIS) score $=1$ and Mini-Cog ${ }^{\mathrm{TM}}$ score $=1$. Cognitive battery provided evidence of severe cognitive decline and hence required further assessment using radiological 
techniques. Patient was advised for PET CT brain to localize areas of brain responsible for cognitive decline.

Findings of PET-CT brain scan performed using a dedicated PET-CT scanner having a 32slice/sec Multidetector Computerized Tomography (MDCT) were observed. Serial axial sections were obtained from skull base to vertex. Patient's blood glucose level at the time of injection was $116 \mathrm{mg} \%$.
PET CT brain demonstrated evidence of age-related cerebral cortical atrophy with mild widening of sulci and a non-dilated ventricular system. Mild diffusely decreased FDG uptake was seen along the bilateral cerebral and cerebellar cortex. Reduced radioactive glucose uptake was noted in bilateral mesial temporal (hippocampal) and parietal regions, predominantly affecting right side with preserved glucose metabolism in sensori-motor cortex, bilateral basal ganglia, thalami and cerebellum. (Figs 1-4)

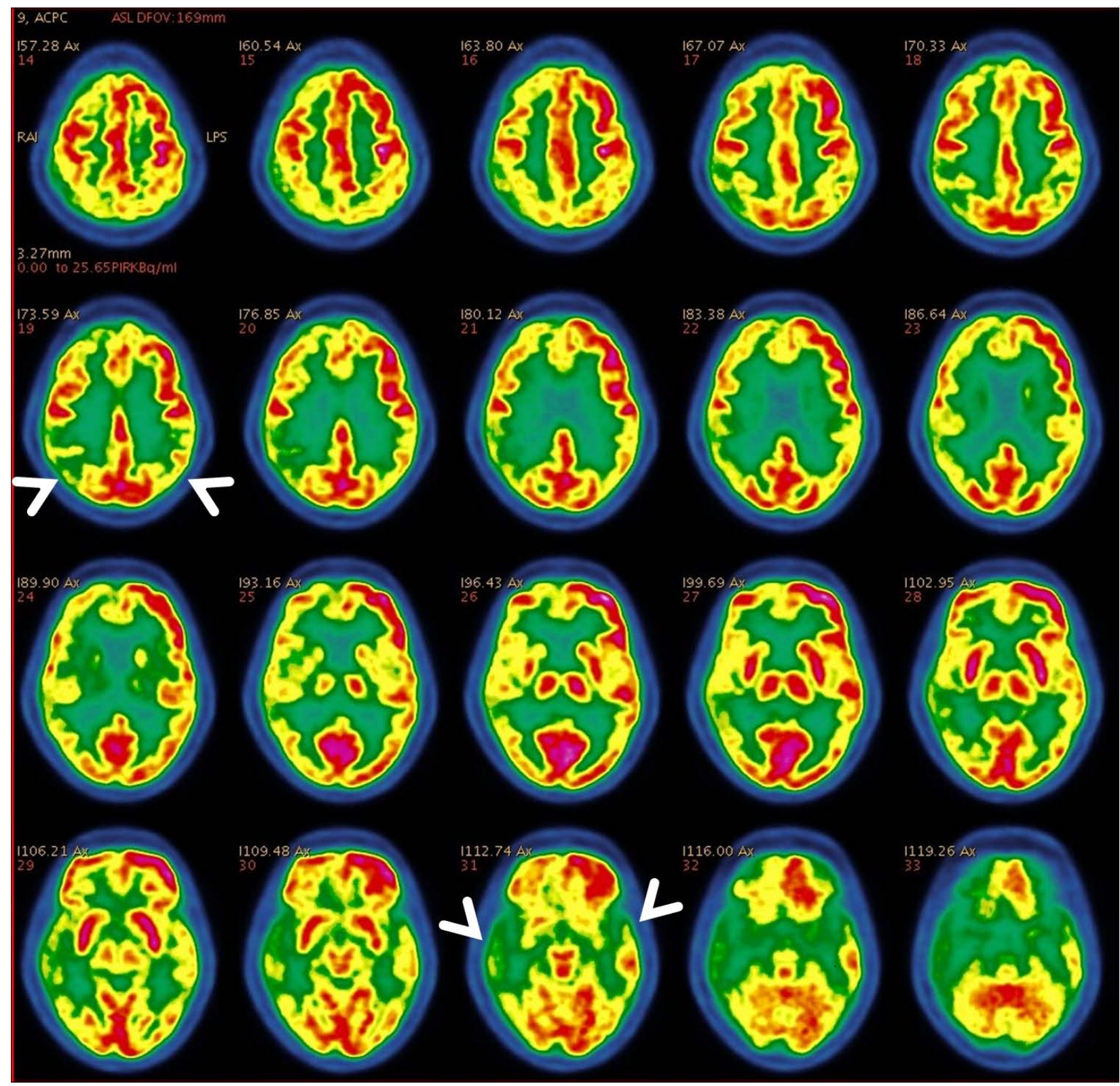

Fig. (1). Axial ${ }^{18}$ F-FDG brain PET slices of a 74-year-old woman with progressive dementia revealed significant FDG hypometabolism involving bilateral parietal cortices and extending to the mesial temporal regions, more predominantly affecting the right side (white arrowheads). Mild hypometabolism involving bilateral anterior cingulated cortices was also seen with preserved glucose metabolism in sensori-motor cortex, bilateral basal ganglia, thalami and cerebellum. These features appear consistent with AD. 


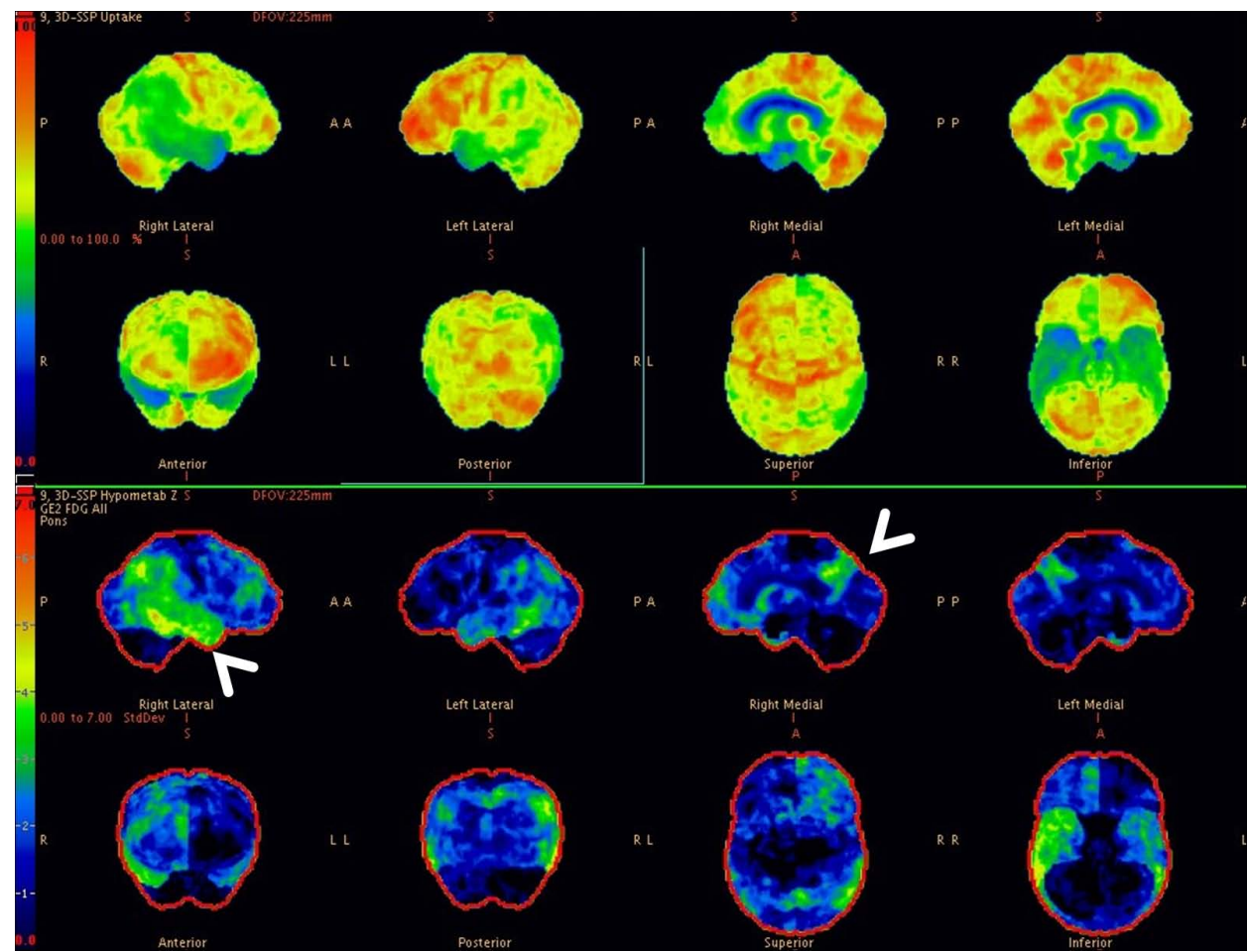

Fig. (2). 3-dimensional stereotactic surface projection images (generated using comparison with a normal standardized brain database, Neuro ${ }^{\mathrm{TM}}$ software package, GE healthcare) showing relative glucose metabolism (top panel) and extent of hypometabolism (lower panel) in standard projections (Right lateral, left lateral, right medial, left medial, anterior, posterior, superior and inferior). These images also confirm the moderate glucose hypometabolism along bilateral parieto-temporal cortices (white arrowheads).

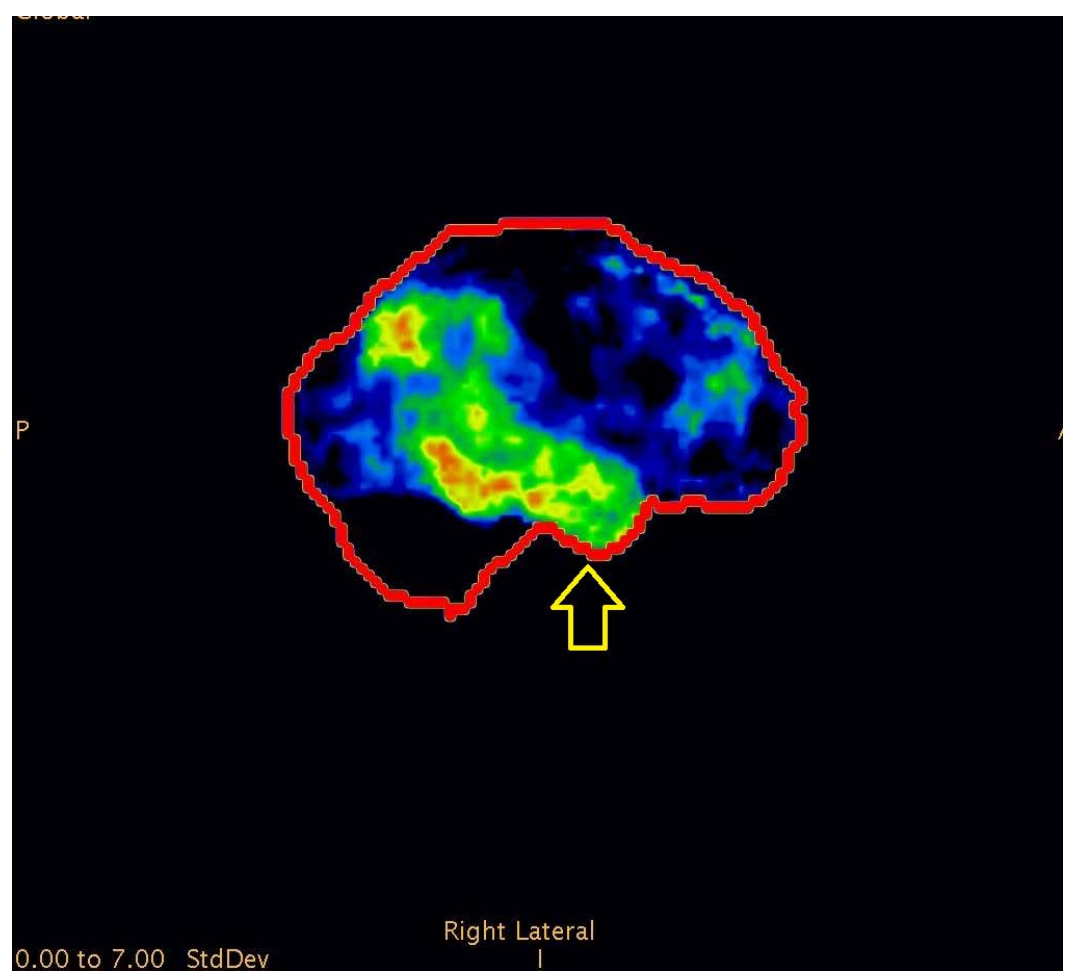

Fig. (3). 3-dimensional stereotactic surface projection images (generated using comparison with a normal standardized brain database, Neuro ${ }^{\mathrm{TM}}$ software package, GE healthcare) showing relative glucose metabolism (top panel) and extent of hypometabolism (lower panel) in standard projections (Right lateral). Current image confirms the moderate glucose hypometabolism (Yellow arrowhead). 


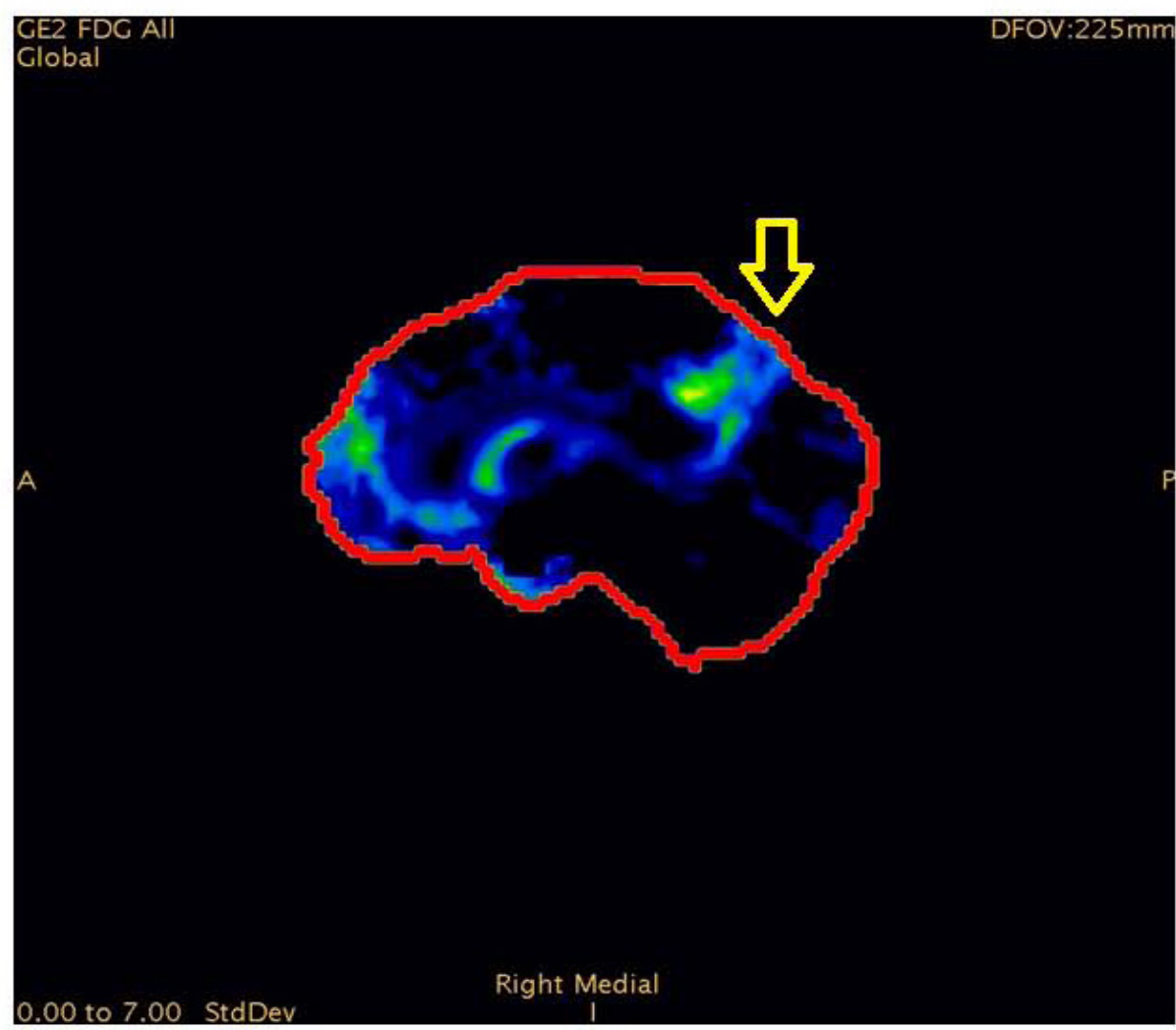

Fig. (4). 3-dimensional stereotactic surface projection images (generated using comparison with a normal standardized brain database, $N$ euro $Q^{\mathrm{TM}}$ software package, GE healthcare) showing relative glucose metabolism (top panel) and extent of hypometabolism (lower panel) in standard projections (Right Medial). Current image confirms the moderate glucose hypometabolism (Yellow arrowhead).

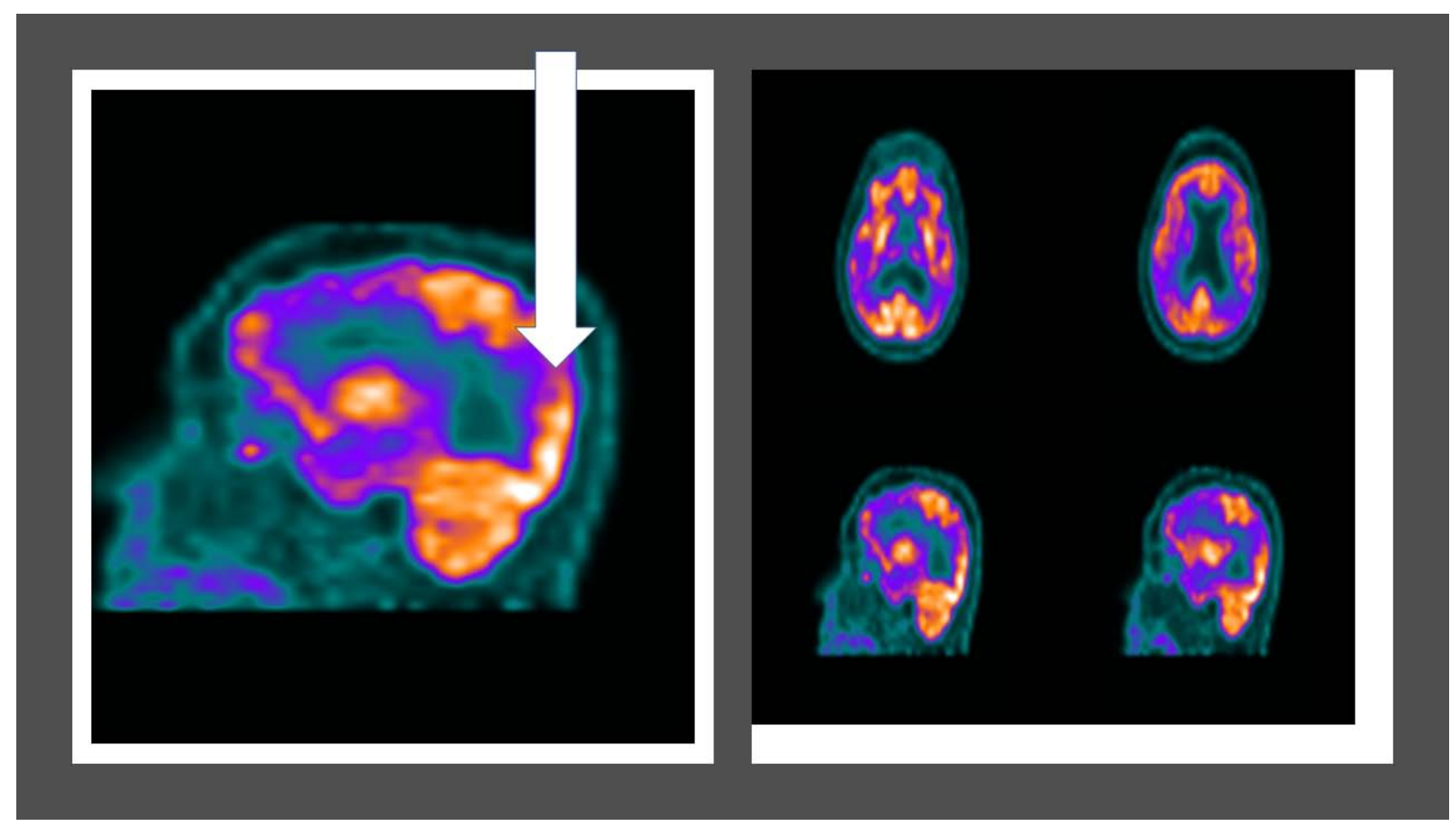

Fig. (5). A 73 year old male with history of 15 years of type 2 diabetes mellitus showing diffuse symmetric hypometabolism in bilateral parietotemporal cortices (White arrow) indicative of Alzheimer's type dementia. 
Another 73 year old type 2 diabetic patient with 15 year history of diabetes and chief complaint of memory loss over last 5 years reported in the hospital was advised PET CT brain for the differential diagnosis of dementia. Cognitive battieries were administered which indicated a severe cognitive decline. PET CT brain indicated diffuse symmetric hypometabolism in the bilateral parieto-temporal cortices (white arrow) indicative of Alzheimer's type dementia (Refer to Fig. 5).

\section{DISCUSSION}

India has been termed as Diabetic capital of the world. A parallel increase in Dementia cases in India in recent years has been observed. ARDSI 2010 report highlights that by 2030, there will be close to 7 million Dementia cases in India. [ARDSI 2010]Global studies have emphasized on association between diabetes and dementia; however, very limited research has been undertaken in the Indian context [Ciudin et al.].

Earlier imaging studies have indicated cortical atrophy in cases of Dementia, although there are limited functional brain imaging studies investigating the association of Dementia and Diabetes [6, 7]. In India, PET CT is typically utilized for the purpose of Oncology diagnosis and prognosis. Very limited utilization of PET CT brain is done for the purpose of Dementia diagnosis and prognosis $[8,9]$.

We observed PET CT brain of one of the Diabetic subjects indicating marked cognitive derangement and the results were indicative of Alzheimer's type pathology. Axial ${ }^{18}$ F-FDG brain PET slices of a 74-year-old woman with progressive dementia revealed significant FDG hypometabolism involving bilateral parietal cortices and extending to the mesial temporal regions, more predominantly affecting the right side. Mild hypometabolism involving bilateral anterior cingulated cortices was also seen with preserved glucose metabolism in the sensori-motor cortex, bilateral basal ganglia, thalami and cerebellum. These features appear consistent with AD (Fig. 1). 3-dimensional stereotactic surface projection images (generated using comparison with a normal standardized brain database, NeuroQ ${ }^{\mathrm{TM}}$ software package, GE healthcare) showed relative glucose metabolism (top panel) and extent of hypometabolism (lower panel) in standard projections (Right lateral (Fig. 3), left lateral, right medial (Fig. 4), left medial, anterior, posterior, superior and inferior). These images also confirm the moderate glucose hypometabolism along the bilateral parieto-temporal cortices (white arrowheads) (Fig. 2).

Both of these case reports hint towards a significant correlation between the poor diabetes control and cognitive derangement as confirmed by the PET CT brain of the patient.

\section{CONCLUSION}

In future, there is a strong need to conduct multiple clinical studies to provide strong evidence for the PET CT brain based association of Diabetes and Cognitive decline.

\section{STANDARDS OF REPORTING}

CARE guidelines have been followed in the study

\section{AUTHORS CONTRIBUTIONS}

Aman Gupta was responsible for Study concept, design, preparation of manuscript, data collection and interpretation. Aashish Gambhir was responsible for setting up Dementia protocol for the PET CT brain and was responsible for the complete assessment of the case based on Nuclear medicine.

\section{CONSENT FOR PUBLICATION}

Not applicable.

\section{FUNDING}

None.

\section{CONFLICT OF INTEREST}

The author declares no conflict of interest, financial or otherwise.

\section{ACKNOWLEDGEMENTS}

Authors hereby acknowledge Sarvodaya Hospital \& Research Center, Faridabad-121006, Delhi NCR, India as an academic research project Clinical site for providing patient pool, administration of cognitive batteries using hospital infrastructure and manpower. We would also like to thank "The Alzheimer's Association" (http://www.alz.org) for allowing the usage of Cognitive batteries used in the study.

\section{REFERENCES}

[1] Alzheimer's and Related Disorders Society of India. The Dementia India Report : Prevalence, impact, costs and services for dementia 2010; 1-10.

[2] Ciudin A, Espinosa A, Simó-Servat O, et al. Type 2 diabetes is an independent risk factor for dementia conversion in patients with mild cognitive impairment. J Diabetes Complications 2017; 31(8): 1272-4. [http://dx.doi.org/10.1016/j.jdiacomp.2017.04.018] [PMID: 28545893]

[3] Cole AR, Astell A, Green C, Sutherland C. Molecular connexions between dementia and diabetes. Neurosci Biobehav Rev 2007; 31(7): 1046-63.

[http://dx.doi.org/10.1016/j.neubiorev.2007.04.004]

[PMID: 17544131]

[4] Cosway R, Strachan MW, Dougall A, Frier BM, Deary IJ. Cognitive function and information processing in type 2 diabetes. Diabet Med 2001; 18(10): 803-10.

[http://dx.doi.org/10.1046/j.1464-5491.2001.00577.x]

[PMID: 11678970]

[5] Croxson SC, Jagger C. Diabetes and cognitive impairment: a community-based study of elderly subjects. Age Ageing 1995; 24(5): 421-4.

[http://dx.doi.org/10.1093/ageing/24.5.421] [PMID: 8669347]

[6] Ewers M, Sperling RA, Klunk WE, Weiner MW, Hampel H. Neuroimaging markers for the prediction and early diagnosis of Alzheimer's disease dementia. Trends Neurosci 2011; 34(8): 430-42. [http://dx.doi.org/10.1016/j.tins.2011.05.005] [PMID: 21696834]

[7] Grinband J, Steffener J, Razlighi QR, Stern Y. BOLD neurovascular coupling does not change significantly with normal aging. Hum Brain Mapp 2017; 38(7): 3538-51.

[http://dx.doi.org/10.1002/hbm.23608] [PMID: 28419680]

[8] Basu S, Kumar R, Alavi A. PET and PET-CT imaging in infection and inflammation: its critical role in assessing complications related to therapeutic interventions in patients with cancer. Indian J Cancer 2010; 47(4): 371-9. 
[http://dx.doi.org/10.4103/0019-509X.73562] [PMID: 21131748]

9] Punit Sharma, Rakesh Kumar, Harmandeep Singh, Sunil Jeph,

Bhagwan Sharma Jai, Kumar Jain Sunesh, et al. 2012 February; Role of FDG PET-CT in detecting recurrence in patients with uterine sarcoma: comparison with conventional imaging Nuclear Medicine Communications 2012 February; 33(2): 185-90.

\section{C) 2020 Gupta and Gambhir.}

This is an open access article distributed under the terms of the Creative Commons Attribution 4.0 International Public License (CC-BY 4.0), a copy of which is available at: (https://creativecommons.org/licenses/by/4.0/legalcode). This license permits unrestricted use, distribution, and reproduction in any medium, provided the original author and source are credited. 